\title{
Justa distribución de beneficios en las declaraciones internacionales de ética en investigación
}

\author{
Ricardo Páez-Moreno, MB, DC.(I)
}

\section{Paez-Moreno R. \\ Justa distribución de beneficios en las declaraciones internacionales de ética en investigación. Salud Publica Mex 20 12;54:637-643.}

\begin{abstract}
Resumen
Varios estándares éticos definidos en las declaraciones sobre investigación biomédica internacional en seres humanos han delineado aquello que es considerado justo en la investigación realizada en países de mediano o bajo ingreso. El cumplimiento de los estándares incide directamente en favor de la salud de los pacientes, quienes se benefician de una mejora de la infraestructura y las capacidades para la investigación en salud locales y en la disminución de la carga de enfermedad. Los beneficios que necesitan ser distribuidos equitativamente pueden clasificarse como: responder a las necesidades o prioridades en investigación locales, dar acceso a beneficios postensayo clínico, propiciar cuidados médicos auxiliares, poner a disposición los nuevos conocimientos, apoyar a los servicios y a la investigación en salud, e incluir en el consentimiento informado qué beneficios potenciales se esperan. Es necesario que aquellas personas relacionadas con la investigación internacional conozcan esta normativa ética y sus posibles beneficios para la salud pública.
\end{abstract}

Palabras clave: justicia; ética; investigación biomédica; declaraciones internacionales

\section{Paez-Moreno R.}

Just sharing of benefits in ethical guidelines

for international research.

Salud Publica Mex 20 I2;54:637-643.

\begin{abstract}
Several ethical standards defined in guidelines for international biomedical research in humans have outlined what is considered just in research conducted in middle to lowincome countries. Compliance with the standards outlined by these guidelines results in direct benefits to the health of the patients, who benefit from improvements in infrastructure, increased local research capacity, and decrease in the burden of disease. In an overall view of the ethical standards, the benefits that need to be distributed equitably can be classified as: addressing the needs and/or priorities of the local research, making post-trial benefits available, fostering ancillary-care benefits, making the new theoretical and practical knowledge available, supporting health services as well as health and ethics research, and including in the informed consent the potential benefits that can be expected. It is necessary that those who deal with international research have knowledge about these ethical guidelines and their potential benefits to public health.
\end{abstract}

Key words: justice; ethics; biomedical research; international acts
$\mathrm{L}$ a justa distribución de beneficios en la investigación internacional llevada a cabo en países de mediano o bajo ingreso ha sido tratada en múltiples declaraciones internacionales de ética y por documentos de comités locales de ética de relevancia mundial. Un aspecto central es el tipo de beneficios que corresponden a las comunidades huéspedes donde se realiza investigación internacional. Su relación con la salud pública es de gran importancia puesto que el número de investigaciones internacionales en los países de bajo o mediano ingreso ha crecido significativamente, y la diferencia en la carga de la enfermedad entre países de alto ingreso y de bajo

(I) Programa de Maestría y Doctorado en Bioética, Universidad Nacional Autónoma de México. México DF. 
ingreso continúa en aumento. ${ }^{1,2}$ Una justa distribución de beneficios redundará no sólo en favor de la salud de los pacientes (p.e. disminución de enfermedades crónicas), sino también en una mejora de la infraestructura y las capacidades para la investigación en salud locales, y por lo tanto en una disminución de la carga de la enfermedad. De ahí la importancia de contar con un adecuado marco ético normativo.

La riqueza de estos pronunciamientos éticos está en ser fruto de grandes consensos logrados y por ello cuentan con una alta estimación moral. Por otra parte, es patente la dificultad de hacer efectivas las recomendaciones dadas por las distintas declaraciones, sobre todo en países de bajo ingreso. ${ }^{3,4}$ No obstante, son un marco imprescindible para hacer buena investigación de manera éticamente aceptable o al menos para tomar conciencia de lo que no se lleva a cabo correctamente y de la necesidad de dar pasos para aproximarse a aquello que es justo.

La abundancia de declaraciones internacionales sobre ética en la investigación internacional en seres humanos ofrece a los comités de ética en investigación, a los investigadores, a las autoridades en salud y a los pacientes certezas para la deliberación bioética sobre la justa distribución de beneficios en los protocolos de investigación internacional en seres humanos. Sin embargo, al ser varias las declaraciones y tocar tantos puntos relativos a la ética de la investigación internacional, es difícil tener claridad sobre la normativa más importante con la que se cuenta para elaborar pronunciamientos bioéticos. Se requiere de una mirada de conjunto que ayude a ver cada uno y la totalidad de recursos éticos con los que se cuenta.

El objetivo de este artículo es ofrecer, para aquéllos que trabajan en la investigación y la salud pública, un panorama sobre la justa distribución de beneficios normada por las principales declaraciones internacionales sobre ética en investigación y los principales temas que abarca, cuya aplicación en la práctica redundará en beneficios para la salud pública y la investigación en salud.

Para esta revisión se ha recurrido a cuatro de las más importantes declaraciones sobre ética en investigación en el ámbito global y a tres de las más relevantes en el plano local (cuyo objeto de estudio es la investigación internacional) provenientes de comités de alta estimación. Se han distinguido y clasificado sus pronunciamientos relacionados con la necesidad de distribuir equitativamente los beneficios producto de la investigación.

Entre las declaraciones internacionales se encuentran las siguientes: 1. La Declaración de Helsinki (DH), realizada por la Asociación Médica Mundial, (AMM). ${ }^{5} 2$. Las Pautas Éticas Internacionales para la Investigación Biomédica en Seres Humanos (normas CIOMS), elaboradas por el Consejo de Organizaciones Internacionales de las Ciencias Médicas. ${ }^{6}$ 3. La Declaración Universal sobre Bioética y Derechos Humanos (DUBDH) confeccionada por la Organización de las Naciones Unidas para la Educación, la Ciencia y la Cultura, (UNESCO). ${ }^{7} 4$. La Declaración sobre los Aspectos Éticos en la Investigación Clínica en los Países en Desarrollo del Grupo Europeo de Ética de las Ciencias y las Nuevas Tecnologías, (GEE). ${ }^{8}$

Entre las declaraciones locales están: 1. El Reporte sobre Políticas y Asuntos Éticos en la Investigación Internacional: Ensayos Clínicos en Países en Desarrollo, elaborada por la antes llamada Comisión Nacional Asesora del Presidente de los EUA(NBAC). ${ }^{9, *}$ 2. El Reporte sobre Ética en Investigación relacionada con la atención de la salud en países en desarrollo, a partir del Nuffield Council on Bioethics (NCB). ${ }^{10}$ 3. La recomendación del Comité Consultor Nacional de Ética para las Ciencias de la Vida y de la Salud de Francia, (CCNE), cooperación en la investigación biomédica entre equipos franceses y equipos de países en vías de desarrollo económico. ${ }^{11}$ Existen otras importantes declaraciones que señalan aspectos importantes para la justa distribución de beneficios, pero que no se toman en cuenta aquí por su carácter más específico o su menor relevancia mundial. Entre ellas están: las pautas para Buenas Prácticas Clínicas promulgadas por la Conferencia Internacional de Armonización, la Declaración sobre Justos Beneficios de la Organización del Genoma Humano, y el Documento Consideraciones Éticas en los ensayos biomédicos preventivos de VIH de la OMS/ONUSIDA.

\section{Discusión}

En este apartado se considerarán, en primer lugar, los principales contenidos en relación con la justa distribución de beneficios en las declaraciones internacionales, y en un segundo momento se clasificarán en siete categorías y se ofrecerá una visión de conjunto.

\section{A. La justa distribución de beneficios en las declaraciones internacionales sobre ética en investigación}

\section{En las declaraciones internacionales}

\section{1) Declaración de Helsinki}

La DH (2008) fue publicada por primera vez en 1964 por la AMM en Finlandia, y es uno de los documentos más utilizados para regular la ética de la investigación. Su quinta revisión fue en el año 2000, y en octubre de 2008 se realizó la última. Los párrafos relacionados con la justa distribución de beneficios en la investigación son el 14,

\footnotetext{
* La NBAC, National Bioethics Advisory Commission, es la actual Comisión del Presidente de EUA para el Estudio de Cuestiones Bioéticas, PCB.
} 
el 17 y el 33. Señalan el deber de que la investigación en poblaciones vulnerables responda a sus necesidades y prioridades de salud, y que existan posibilidades razonables de que la población pueda beneficiarse de sus resultados. El párrafo 33 señala que al concluir el estudio, los pacientes reclutados tienen derecho a ser informados sobre el resultado del estudio y a disfrutar de cualquier beneficio que resulte de éste.*

2) Pautas Éticas Internacionales para la Investigación Biomédica en Seres Humanos

En la pauta cinco se menciona la información esencial para potenciales sujetos de investigación: si se proporcionará dinero $\mathrm{u}$ otras formas de bienes materiales por la participación del individuo; qué beneficios directos se esperan para los sujetos que participan en la investigación, en caso de haberlos; qué beneficios se espera de la investigación para la comunidad o sociedad en general, o su contribución al conocimiento científico; si cualquier producto o intervención de efectividad y seguridad comprobadas por la investigación estará a disposición de los sujetos después de haber completado su participación en la investigación, cuándo y cómo estará disponible, y si se espera que paguen por él.

La revisión 2002 se promulgó pensando en los países en desarrollo, al reiterar el requisito ya estatuido en la revisión de 1993 de que la investigación se haga responsable de las necesidades de salud y las prioridades de la comunidad en que se lleva a cabo (párrafo 10). Se eleva a nivel de pauta dicho párrafo, en vez de ser un comentario como lo era en la edición de 1993, y se afirma que cualquier intervención o producto desarrollado o conocimiento generado estará disponible razonablemente para beneficio de aquella población o comunidad.

Finalmente, las pautas 20 y 21 señalan la obligación ética de los patrocinadores externos de contribuir a la capacidad local de crear investigación biomédica, evaluación ética y supervisión; también de proporcionar servicios para la atención de la salud.

\section{3) Declaración Universal sobre Bioética y los Derechos Humanos (DUBDH) de la UNESCO}

El 19 de octubre de 2005, la UNESCO publicó la DUBDH. En este documento se aborda la justicia en la investigación multinacional con mayor especificidad. Se incluyen preocupaciones sobre la justicia desde los países de bajo

\footnotetext{
* En octubre de 2008 ha tenido lugar una última revisión de la Declaración, la cual cambió el párrafo 19 por el 17 y el párrafo 30 por el 33, este último con una redacción más general, quitando todo compromiso de la investigación a otorgar los mejores métodos de profilaxis, diagnóstico o tratamiento identificados a través del estudio.
}

ingreso, la necesidad de compartir los beneficios de las investigaciones internacionales, así como cuestiones de simetría y justicia en la investigación internacional en seres humanos. El artículo 15 es el que da pautas más concretas sobre el tipo de beneficios a compartir: asistencia especial y duradera a las personas y los grupos que hayan tomado parte en la actividad de investigación y reconocimiento de los mismos; acceso a una atención médica de calidad; suministro de nuevas modalidades o productos de diagnóstico y terapia obtenidos gracias a la investigación; apoyo a los servicios de salud; acceso a los conocimientos científicos y tecnológicos; e instalaciones y servicios destinados a crear capacidades en materia de investigación.

4) Punto de vista del Grupo Europeo de Ética de las Ciencias y las Nuevas Tecnologías

El Grupo Europeo de Ética de las Ciencias y de las Nuevas Tecnologías (GEE) es un órgano consultivo independiente y multidisciplinario de la Comisión Europea. Señala lo siguiente: (párrafo 1.25) la legitimidad de un ensayo clínico se logra en tanto tenga en cuenta las prioridades de salud de los participantes y su interés previsible por mejorar los servicios de salud en el país huésped; (1.26) el primer efecto positivo de un ensayo clínico sobre los servicios de salud es la generación de conocimiento, el cual debe compartirse con aquéllos donde surge el nuevo conocimiento, el acceso a nuevos tratamientos, y el progreso de las capacidades de desarrollo inducido por la realización del ensayo; (2.13) la disponibilidad del tratamiento después del ensayo ha de garantizarse, como en los países ricos, hasta que los servicios públicos de salud puedan asegurar el medicamento para el enfermo, aún si el medicamento se necesita de por vida. Y para que los pacientes no se queden desprovistos del beneficio generado -hasta que la medicina pública lo haga suyo-habrán de emitirse licencias obligatorias. ${ }^{12, *}(2.14)$ Los resultados del ensayo han de ser comunicados a

\footnotetext{
* El tratado Agreement on Trade-Related Aspects of Intellectual Property Rights (TRIPS) emitido por la Organización Mundial del Comercio para proteger los derechos de propiedad intelectual bajo leyes internacionales comunes, permite las llamadas licencias obligatorias o "compulsory licenses" para facilitar el acceso de medicamentos patentados a países de bajo ingreso. Estas son licencias acordadas entre el propietario de la patente y cierto país para determinados usos y modos de utilización. El monto de las licencias a menudo es establecido por la legislación local o a veces se define a través de una negociación. La Declaración de Doha de la OMC sobre el TRIPS y la Salud Pública en el año 2001 tuvo la finalidad de no impedir medidas de salud pública por motivo del TRIPS. Desde entonces, sólo se han detectado 24 licencias obligatorias en 17 países, más de la mitad de ellos de ingreso medio o alto. Llama la atención que México no ha solicitado ninguna. A partir de 2006 ha declinado sustancialmente la petición de licencias obligatorias.
} 
los médicos, pacientes e investigadores asociados, sean positivos o negativos. Los nuevos conocimientos han de ponerse al alcance de la comunidad científica del país y del conjunto de la población. Ha de tomarse en cuenta a los médicos e investigadores locales en la repartición de derechos de propiedad industrial.

\section{Declaraciones del ámbito local}

1) Reporte sobre Políticas y Asuntos Éticos en la Investigación Internacional: Ensayos Clínicos en Países en Desarrollo, de la NBAC

El documento de la NBAC hace múltiples recomendaciones respecto a las investigaciones internacionales en países en desarrollo. En el sumario ejecutivo (Recomendación 1.3), se recomienda que los ensayos clínicos deben responder a las necesidades en salud de los países huéspedes, (2.3) prever cómo incluir a los representantes de las comunidades en el diseño e implementación de los protocolos, (3.3) e incluir en el consentimiento informado qué beneficios potenciales para los participantes se esperan al finalizar el estudio. (4.1) Respecto a disponer de los beneficios para los participantes, recomienda que una vez terminados los ensayos, debe preverse y hacerse público qué harán los investigadores para que todos los participantes tengan acceso al producto médicamente benéfico después de la conclusión del proyecto, explicitando la duración, extensión y financiamiento de dicho beneficio. En caso de que no se negocien acuerdos previos, se deberá justificar al comité de ética de investigación la razón de tal negativa. (4.2) Los investigadores deben prever antes de la iniciación del proyecto cómo se pondrán razonablemente a disposición los beneficios de las investigaciones exitosas para la comunidad o el país entero; si no se prevé un beneficio para la población huésped, habrá de justificarse la razón ante el comité de ética de investigación. (4.3) Cuando sea posible, habrán de acordarse los beneficios producto de la investigación $\mathrm{u}$ otros beneficios que podrán derivarse para la comunidad, previamente al inicio del protocolo. (5.6 y 5.7) Los patrocinadores e investigadores extranjeros deben apoyar la capacidad de investigación local y de ética en investigación.

2) Reporte sobre Ética en Investigación Relacionada con la Atención de la Salud en Países en Desarrollo del Nuffield Council of Bioethics

(Párrafo 2.30) La importancia de que la investigación tenga que ver con las prioridades locales en investigación; (5.26) la capacitación y entrenamiento de todos los involucrados en ética de la investigación; (8.20) y a los comités de ética en investigación; (6.5) informar en el consentimiento informado sobre los posibles riesgos y beneficios; (9.12) fortalecer la capacidad en investigación y atención de la salud locales orientadas a que se sostengan una vez que termine la investigación; (9.24) prever la intervención exitosa al grupo control una vez que termine el protocolo; (9.31) prever dar el tratamiento exitoso a los participantes al final del ensayo clínico; (9.48) y evaluar la posibilidad de extender el tratamiento exitoso a toda la comunidad; (9.49) e incluso alguna o la totalidad de la población del país y en su defecto justificar por qué es necesario aun así hacer la investigación; (9.52) por último, la necesidad de desarrollar expertise en investigación local de tal manera que el equipo obtenido pueda ser debidamente utilizado y mantenido.

3) Recomendación del CCNE: la cooperación en la investigación biomédica entre equipos franceses y equipos de países en vías de desarrollo económico

Los equipos de investigación franceses han de identificar las particularidades sociales, culturales, políticas y religiosas, capaces de impedir la investigación o de llevar a cabo un buen desempeño de la misma (párrafo 2); los resultados del protocolo deben hacerse accesibles a la comunidad científica y al público (5).

\section{B. Principales temas abordados por las declaraciones en relación con la justa distribución de beneficios en las investigaciones internacionales}

\section{Responder a las necesidades o prioridades en investigación locales}

Es la recomendación más repetida, presente en todas las declaraciones. Las normas CIOMS piden que desde antes de iniciar la investigación se hagan todos los esfuerzos para que esto suceda. Sin embargo, a pesar de la obviedad de esta exigencia, la investigación internacional se caracteriza en buena medida por responder a las necesidades del mercado, por ejemplo priorizando la investigación sobre las llamadas life style drugs que son medicamentos para tratar síntomas producidos por el estilo de vida apresurado y consumista contemporáneo, tales como la obesidad o aliviar los síntomas del envejecimiento, o para generar me too drugs que son moléculas a las que se les han realizado pequeños cambios farmacológicos para lograr la aprobación de una nueva patente y ganar nuevos nichos de mercado. ${ }^{13,14}$ Por otra parte, las grandes necesidades en salud de la mayoría de la población mundial continúan desatendidas respecto de investigación, lo que origina el coeficiente 10/90, es decir, de los 70 millones 
de dólares gastados anualmente en el mundo para la investigación por los sectores público y privado, sólo 10\% sirven para la resolución de los problemas de salud de 90\% de la población mundial; por el contrario, $90 \%$ del gasto en investigación está destinado a las enfermedades que afectan a $10 \%$ de la población mundial. ${ }^{15}$

\section{Dar acceso a beneficios postensayo clínico}

El tener acceso, cuando menos para los sujetos partipantes, a intervenciones benéficas producto de la investigación, o también llamados beneficios postensayo clínico (post-trial obligations) -para las normas CIOMS, que los frutos de la investigación estén disponibles razonablemente, lo cual ha sido objeto de diversas críticas ${ }^{16}{ }^{16}$ es la manera más concreta de distribuir beneficios producto de la investigación y que ha sido origen de largos e intensos debates, tales como el del párrafo 30 de la revisión anterior de la DH. ${ }^{*}$ Con este tipo de beneficios se busca evitar que los investigadores o patrocinadores provenientes de un país de alto ingreso se marchen del país huésped una vez finalizado el protocolo y abandonen a los pacientes que aún necesitan un tratamiento que ha demostrado ser efectivo; esto último es lo que se ha llamado "investigación safari."17 Las declaraciones señalan distintas maneras de proporcionar el acceso a los productos exitosos. La DUBDH pide el suministro de nuevas modalidades o productos de diagnóstico y terapia obtenidos gracias a la investigación, así como una asistencia especial y duradera a los participantes. El NCB requiere el acceso a intervenciones benéficas, razonablemente disponibles, para la comunidad o el país entero, y la NBAC que éste se establezca antes de iniciar el proyecto. La $\mathrm{DH}$ pide que el protocolo describa los arreglos para el acceso a beneficios después del ensayo. La DH y las normas CIOMS solicitan el acceso a otros cuidados o beneficios apropiados.

\section{Propiciar cuidados médicos auxiliares}

Consiste en propiciar el acceso a atención médica por alguna enfermedad diagnosticada durante el curso de una investigación y no relacionada con esta última. $\mathrm{Al}$ encontrarse con otra enfermedad al hacer la investigación ¿existe el deber de tratarla por parte de los investi-

\footnotetext{
* El párrafo 30 pedía dar acceso a los mejores métodos de profilaxis, diagnóstico o tratamiento identificados a través del estudio; el actual párrafo 33 habla sólo de dar acceso a cualquier beneficio que resulte de la investigación.
}

gadores? Hay quienes señalan el deber ético de hacerlo por las grandes necesidades en salud de los países de bajo ingreso, y hay quienes niegan cualquier tipo de obligación puesto que los investigadores son tales y no médicos. A estos cuidados médicos se les ha llamado beneficios auxiliares (ancillary-care responsibilities) respecto del principal beneficio fruto de la investigación. En bioética son aquellos beneficios que van más allá de los requerimientos de validez científica, seguridad, guardar las promesas o rectificar daños en una investigación. ${ }^{18}$ Comprendería dar seguimiento a diagnósticos encontrados por los exámenes propios del ensayo clínico o tratar males no relacionados con los fines del ensayo clínico. Existe poca referencia a estos cuidados en las declaraciones internacionales. Las normas CIOMS (pauta 21) son las que más dicen algo al respecto. Sin embargo, es un tema debatido en ética de la investigación, ante el cual se han decantado diversas posturas y cursos de acción. ${ }^{19}$

\section{Poner al alcance de la comunidad científica local los nuevos conocimientos teóricos y prácticos}

Se trata de un aspecto crucial en la realización de la investigación biomédica internacional, pero al mismo tiempo es un tema difícilmente aceptado que implica ir en sentido contrario al sistema global de patentes TRIPS. En el fondo, la transferencia de conocimientos implica enseñar a las comunidades de investigadores huéspedes a saber cómo y no sólo utilizarlas como subsedes de una investigación diseñada, procesada y controlada desde los centros de investigación o la industria farmacéutica de países de alto ingreso. Al transferir conocimientos se trabaja en favor del fortalecimiento de los centros de investigación periféricos en vistas a producir medicamentos a un precio accesible, lo cual va en contra de la dependencia que favorece la investigación internacional bajo el sistema global de patentes, y que ha sido calificada como "feudalismo informacional." Prueba de esto último es el citado TRIPS que ha buscado impedir la copia de medicamentos en caso de pandemias como el sida, por ejemplo en Sudáfrica, Brasil e India. Las batallas se han ganado gracias a la postura de los gobiernos locales y la presión de la opinión pública. ${ }^{20}$ Por otra parte, los proyectos de investigación ya vienen diseñados desde el extranjero, y a los investigadores locales no se les dan condiciones de igualdad; es más, cuando son exitosos los resultados, en la publicación muchas veces no se les incluye como coautores de la investigación. Todo lo anterior representa una gran inequidad en la investigación internacional. Las declaraciones DUBDH, el GEE, y el CCNE señalan la necesidad de una transferencia de conocimientos. 


\section{Apoyar a los servicios en salud, investigación en salud y} ética de la investigación

Se refiere a otra medida de justicia que puede ser vista desde la justa compensación que merecen los centros de salud locales por albergar una investigación extranjera, $o$ la justa inversión en salud que requieren las instituciones de poblaciones vulnerables o sitios con escasos recursos donde se realizan investigaciones. En el primer caso, relacionado con la justicia conmutativa (se refiere a la igualdad o equilibrio entablados entre dos contratantes), los beneficios con que se compensa a las instituciones locales por el uso de instalaciones, personal, equipo, y por el reclutamiento de pacientes, son mucho menores a los enormes dividendos que obtiene la industria farmacéutica por la aprobación de la patente de algún producto exitoso y al ahorro obtenido por realizar sus investigaciones en países de bajo ingreso y con gran cantidad de pacientes. ${ }^{21}$ En el segundo caso, se refiere a aquellos lugares afectados por múltiples determinantes sociales de la salud ${ }^{*}$ donde la justicia social pide que las investigaciones tengan que ver con las grandes necesidades en salud de la población y colaboren con solucionarlas. El apoyo a los servicios de salud, a la capacidad en investigación en salud y el entrenamiento en ética de la investigación es una manera concreta de ayudar a revertir determinantes sociales que merman la atención de la salud de localidades donde se realizan investigaciones internacionales. De diversas maneras, esta obligación es señalada por las normas CIOMS, la DUDBH, el GEE, la NBAC y el NCB.

6. Incluir en el consentimiento informado qué beneficios potenciales se esperan para los pacientes al finalizar el estudio

Se trata de un aspecto de total relevancia señalado por la NBAC, el NBC y las normas CIOMS (que piden que sean beneficios directos para la comunidad y la sociedad en su conjunto), puesto que los pacientes son los receptores directos de los beneficios producto de la investigación, y es necesario contar con su debida anuencia. En el consentimiento informado de los ensayos clínicos internacionales muchas veces viene incluido que el protocolo beneficiará a la ciencia, a futuros pacientes, $\mathrm{o}$ a los voluntarios a través de la supervisión médica que

\footnotetext{
* Son las circunstancias en que las personas nacen, crecen, viven, trabajan y envejecen, incluido el sistema de salud. Esas circunstancias son el resultado de la distribución de la riqueza, el poder y los recursos a nivel mundial, nacional y local, que depende a su vez de las políticas adoptadas. Algunos ejemplos son la atención insuficiente de la salud en cantidad y calidad, la pobre educación, la desigualdad social, la violencia, el hacinamiento, etc.
}

recibirán, o el posible mejoramiento de su padecimiento con el tratamiento experimental. Pero es de llamar la atención que en ningún caso en una investigación sobre protocolos internacionales en México se les ofreció a los pacientes acceso al medicamento en caso de ser exitoso. Este dato es preocupante, y muestra la distancia existente entre la claridad de las declaraciones internacionales y la práctica. ${ }^{3}$

En el cuadro I se muestran de manera conjunta los distintos tipos de beneficios señalados por las diversas declaraciones internacionales.

\section{Conclusiones}

Las declaraciones internacionales sobre ética en investigación señalan una serie de beneficios que por motivos de justicia corresponde a los patrocinadores de los protocolos internacionales otorgar a los pacientes, comunidades e instituciones locales. Entre estos están los que redundan en bien directo de los pacientes, como los beneficios postestudio y los beneficios de cuidados médicos auxiliares, y los beneficios a la infraestructura sanitaria, de investigación en salud, de ética en investigación, incluida la transferencia de conocimientos. Estos tipos de beneficios repercuten positivamente en una disminución de la carga de la enfermedad a nivel local e inciden en una mejora de la salud pública. Es necesario que los comités de ética en investigación, las autoridades de cada institución en salud -desde el director del hospital hasta el jefe de investigación-, los investigadores locales y los pacientes, estén familiarizados con esta normativa ética para tomar conciencia de aquello que en justicia se puede exigir, rechazar aquellos protocolos que no la cumplan, y fomentar así que la investigación internacional colabore efectivamente en un bienestar compartido.

Declaración de conflicto de intereses. El autor declaró no tener conflicto de intereses.

\section{Referencias}

I. Glickman S, McHutchison J, Peterson E, Cairns Ch, Harrington R, Califf $\mathrm{R}$, et al. Ethical and scientific implications of the globalization of clinical research. N Engl JMed 2009;360:816-823.

2. Macklin R. Research multinational. En: Post S, comp. Encyclopedia of bioethics. 3a. ed. Nueva York: Mac Millan, 2004;4:2347-2357.

3. Páez R, García de Alba J. International research and just sharing of benefits in Mexico. Dev World Bioeth 2009;(9):65-73.

4. Cohen E, O'Neill J, Joffres M, Upshur R, Mills E. Reporting ethics issues in registered trials. Dev World Bioeth 2009;(9):74-80.

5. Asociación Médica Mundial. Declaración de Helsinki [monografía en internet]. Ferney-Voltaire: AMM, 2008 [consultado: 2012 enero 25]. Disponible en: http://www.wma.net/es/30publications//Opolicies/b3/I7c_es.pdf. 6. Council for International Organization of Medical Sciences. International ethical guidelines for biomedical research involving human subjects 


\section{Cuadro I}

\section{VISIÓN DE CONJUNTO DE LOS ESTÁNDARES ÉTICOS PARA UNA JUSTA DISTRIBUCIÓN DE BENEFICIOS EN LAS DECLARACIONES INTERNACIONALES SOBRE ÉTICA EN INVESTIGACIÓN}

\begin{tabular}{|c|c|c|c|c|c|c|}
\hline \multirow{3}{*}{$\begin{array}{l}\text { Declaración } \\
\text { internacional }\end{array}$} & \multicolumn{6}{|c|}{ Estándares éticos requeridos para las investigaciones internacionales } \\
\hline & Responder $a$ & Acceso a & Beneficios auxiliares & Acceso & Apoyo a servicios & Incluir tipo de beneficios \\
\hline & necesidades $y$ & intervenciones & de atención & a nuevos & e investigación & en el consentimiento \\
\hline & prioridades locales & benéficas & de la salud & conocimientos & en salud y ética & informado \\
\hline
\end{tabular}

\begin{tabular}{lllll}
$\mathrm{DH}^{\prime}$ & $\sqrt{ }$ & $\sqrt{ }$ & $\sqrt{ }$ & $\sqrt{ }$ \\
\hline $\mathrm{ClOMS}^{2}$ & $\sqrt{ }$ & $\sqrt{ }$ & $\sqrt{ }$ & $\sqrt{ }$ \\
\hline $\mathrm{DUBDH}^{3}$ & $\sqrt{ }$ & $\sqrt{ }$ & $\sqrt{ }$ & $\sqrt{ }$ \\
\hline $\mathrm{GEE}^{4}$ & $\sqrt{ }$ & $\sqrt{ }$ & $\sqrt{ }$ & $\sqrt{ }$ \\
\hline $\mathrm{NBAC}^{5}$ & $\sqrt{ }$ & $\sqrt{ }$ & $\sqrt{ }$ & $\sqrt{ }$ \\
\hline $\mathrm{NCB}^{6}$ & $\sqrt{ }$ & $\sqrt{ }$ & $\sqrt{ }$ & $\sqrt{ }$
\end{tabular}

Los primeros cuatro documentos representan declaraciones internacionales. Si bien los últimos tres son documentos locales en cuanto a su autoría, su objeto de estudio es la investigación desarrollada entre países en el ámbito internacional.

I Declaración de Helsinki

2 Pautas Éticas Internacionales para la Investigación Biomédica en Seres Humanos

3 Declaración Universal sobre Bioética y los Derechos Humanos de la UNESCO

${ }^{4}$ Punto de Vista del Grupo Europeo de Ética de las Ciencias y las Nuevas Tecnologías

5 Reporte sobre Políticas y Asuntos Éticos en la Investigación Internacional: Ensayos Clínicos en Países en Desarrollo, de la National Bioethics Advisory Commission

6 Reporte sobre Ética en Investigación Relacionada con la Atención de la Salud en Países en Desarrollo del Nuffield Council of Bioethics

7 Recomendación del Comité Consultatif National d'Ethique: la cooperación en la investigación biomédica entre equipos franceses y equipos de países en vías de desarrollo económico. México DF, febrero de 2012

[monografía en internet]. Geneva: CIOMS, 2002 [consultado: 2012 enero 25]. Disponible en: http://www.paho.org/Spanish/BIO/CIOMS.pdf. 7. United Nations Educational Scientific and Cultural Organization. Universal declaration on bioethics and human rights [monografía en internet]. Paris: UNESCO, 2005 [consultado: 2012 enero 25]. Disponible en: http://portal.unesco.org/en/ev.php-URL_ID=3 1058\&URL_DO=DO_ TOPIC\&URL_SECTION=20I.html.

8. Groupe Européen d'Ėthique des Sciences et des Nouvelles Technologies auprès de la Commission Européenne. Aspects ethiques de la recherche clinique dans les pays en developpement. Bruxelles: GEE, 2003 [consultado: 2012 enero 25]. Disponible en: http://ec.europa.eu/bepa/ european-group-ethics/docs/cp 17_fr.pdf.

9. National Bioethics Advisory Commission. Ethical and policy issues in international research: clinical trials in Developing Countries [monografía en internet]. Bethesda, MD: NBAC, 200I [consultado: 2012 enero 25]. Disponible en: http://bioethics.georgetown.edu/nbac/clinical/ExecSum.pdf. 10. Nuffield Council of Bioethics. The ethics of research related to care in developing countries [monografía en internet]. London: NCB, 2002 [consultado: 2012 enero 25]. Disponible en: http://www.nuffieldbioethics. org/sites/default/files/Ethics\%20of\%20research\%20related\%20to\%20 healthcare\%20in\%20developing\%20countries\%20l.pdf.

II. Comite Consultatif National d'Ethique pour les Sciences de la Vie et de la Sante. La coopération dans le domaine de la recherche biomédicale entre équipes françaises et équipes de pays en voie de développement économique. Avis No. 41 [monografía en internet]. Paris: CCNE, 1993 [consultado: 2012 enero 25]. Disponible en: http://www.ccne-ethique.fr/ docs/fr/avis04I.pdf.

12. Beall R, Kuhn R. Trends in compulsory licensing of pharmaceuticals since the Doha Declaration: a database analysis. PLoS Med [serie en internet] 2012 [consultado 2012 febrero 3]; 9: [aprox. 9 Pp]. Disponible en: http://www.plosmedicine.org/article/info\%3Adoi\%2FI0.1371\%2Fjournal. pmed. 1001154 .
13. Cahoon L. Companies throw their weight behind new antiobesity drugs. Nat Med 2010;16:136.

14. Páez R. La investigación de la industria farmacéutica: ¿condicionada por los intereses del mercado? Acta Bioeth [serie en internet] $201 \mathrm{I}$ [consultado 2012 febrero 3]; 17: 237-246. Disponible en: http://www.scielo.cl/ pdf/abioeth/v17n2/al0.pdf.

15. Global Forum for Health Research. 10/90 Report on Health Research 2003/2004 [monografía en internet]. Geneve: GFHR, 2004 [consultado: 2012 enero 25]. Disponible en: http://bibliotecas.salud.gob.mx/gsdl/collect/publin I/index/assoc/HASHcle3.dir/doc.pdf

16. The Participants in the 200 I Conference on Ethical Aspects of Research in Developing Countries. Moral Standards for Research in Developing Countries. From "Reasonable Availability" to "Fair Benefits." Hastings Cent Rep 2004;34:17-27.

17. Macklin R. Ética de la investigación internacional. El problema de la justicia hacia los países menos desarrollados. Acta Bioeth [serie en internet] 2004 [consultado 2012 febrero 3]; 10:27-35. Disponible en: http:// www.scielo.cl/pdf/abioeth/v10nl/art04.pdf.

18. Belsky L, Richardson H. Medical researchers' ancillary clinical care responsibilities. BMJ 2004;328: 1494-1496.

19. Participants in the 2006 Georgetown University Workshop on the Ancillary-Care Obligations of Medical Researchers Working in Developing Countries. The ancillary-care obligations of medical resarchers working in developing countries. PLoS Med [serie en internet] 2008 [consultado 2012 febrero 3]; 5: 709-7/3. Disponible en: http://www.scielo.cl/ pdf/abioeth/vIOnl/art04.pdf.

20. Drahos P, Braithwaite J. Informational feudalism. London: Earthscan Publications, 2002:I-I8.

2I. Ballantyne A. Benefits to research subjects in international trials: do they reduce exploitation or increase undue inducement? Dev World Bioeth 2008;8:178-191. 